\title{
Improvement to Stress Calculation Model of LRM in Thick plate
}

\author{
LIAO Kai ${ }^{1,2, a}$, LI Li-jun ${ }^{1, b}$, WU Yun-xin ${ }^{3, c}$, GONG Hai ${ }^{3, d}$ \\ 1. School of Mechanical and Electrical Engineering, Central South University of Forestry and \\ Technology, Changsha 410083, China \\ ${ }^{2}$.Guilin University of Electronic Technology, Guilin, China \\ ${ }^{3}$.School of Mechanical and Electronic Engineering, Central South University, Changsha, China. \\ a44806474@qq.com; b1518723648 @qq.com; 'wuyunxinatcsu @qq.com; 40835743 @qq.com
}

Keyword: LRM; amended function; deformation error; stress measurement

Abstract: Layer removal method is an experiment method which is used to calculate the average stress indirectly by deformation of the specimen. However, in experiments, some certain predeformation can be caused after each clamping, which can affect the accuracy of the test data, and make the deformation data larger. Based on the comparison of the simulation calculation, this paper builds an amended function by fitting the deformation error, which solves the deviation caused by larger deformation on stress calculation while the specimen is milled by layer, that is to say, the stress calculation model can be compensated by this function. This method can make the experimental calculation model more accurate.

\section{Introduction}

Layer removal method is a more traditional mechanical testing method. With the development of strain gauge test technology, mathematical model of LRM is improved. Daniel James Greving put forward an improved algorithm (MLRM) for hot coating material in the paper[1], which was to assume that stripped layer stress was a constant based on the principle of superposition. He solved the corresponding stress of the deformation layer by layer, and made the successive residual stress distribution come to be matching results by dispersing it to each strain interval. GONG H[2,3] used the methods above to make researches on the residual stress of aluminum alloy thick plate of full thickness. The essence of this algorithm should belong to be discrete method assuming that the stress is a constant within a certain layer depth along the thick plate. At the same time, Virkar, Michael B Prime put forward that integral method can be applied to solve the problem of thick plate stress calculation and error analysis[4,5], in which the principle is to structure stress distribution of thick plate by a hypothetical continuous function, and then the curve solved has better matching degree. In the discussion of these methods, however, they did not discuss the pre-deformation (bending deformation caused by clamping force ) caused by repeatedly clamping, or treated it as a negligible factor. In fact, this omission may lead the experiment of stress to be generally larger, because the deformation data is not normally increasing. According to the deformation within the elastic range, we can amend it by compensating the deformation error.

\section{The original calculation model}

The first category integral form presented by Gary S. Schajer [6]can describe the relationship of the stress-strain, that is the integral method[5], this method solves a continuous change curve of the stress distribution with discrete experiment data. 


$$
\varepsilon(t)=\int_{0}^{t} f(H, t) \sigma(H) d H
$$

$t$ - thickness of stripping layer; $\sigma(H)$ —stress of the thickness $\mathrm{H} ; \varepsilon(t)$ - the measured strain; $f(H, t)$ - sensitive function of deformation in the stress of thickness $\mathrm{H}$, is kernel function, whose result is associated with cutting depth and sample size.

Literature [7] deduced this kind of integral method, and acquired the LRM experimental stress calculation model of thick plate. Residual stress can be supposed using polynomial $P=\left(\frac{z}{H}\right)^{i}$, and result is found solving the undetermined coefficient $A_{i}$ and $B_{i}$,

$$
\sigma_{x}(z)=\sum_{i=0}^{n} A_{i} P_{i} ; \sigma_{y}(z)=\sum_{j=0}^{m} \mathrm{~B}_{j} P_{j} .
$$

Combining the equations above mentioned, the undetermined coefficients can be solved, because of the rule and forms of planer stress distribution are similar, so the same order polynomial can be approximate.

$$
\varepsilon_{x}(t)=\frac{2}{E} \sum_{i=0}^{n} \frac{\left(A_{i}-v B_{i}\right)(i-1)}{(i+1)(i+2)}(1-\alpha)^{i} ; \quad \varepsilon_{y}(t)=\frac{2}{E} \sum_{i=0}^{n} \frac{\left(B_{i}-v A_{i}\right)(i-1)}{(i+1)(i+2)}(1-\alpha)^{i}
$$

Among them $\alpha=\frac{t}{H}$ is the normalized thickness, dimensionless $。$ The number of undetermined coefficients is $2 i$, number of layer peeling $m$, the matrix type:

$$
\begin{aligned}
& \left\{\varepsilon_{m}\right\}=\left[c_{m 1}, c_{m 2} \ldots c_{m i}\right] \cdot\left\{A_{i}-v B_{i}\right\} . \\
& \left\{A_{i}-v B_{i}\right\}=\left[[C]^{T}[C]\right]^{-1}[C]^{T}\left\{\varepsilon_{m}\right\} .
\end{aligned}
$$

Matrix $[C]$ is $m \times i$, combining the longitudinal and lateral strain data the $A_{\mathrm{i}}, B_{i}$ can be calculated.

\section{The experiment error}

Strain reflects the objective deformation, and also determines the accuracy of the stress calculation. Due to the particularity of layer removal conditions, the calculation result and actual result has certain deviation, which is mainly caused by the difference of the measured deformation and deformation theory. Firstly, the vise of the clamping can cause certain pre-deformation in the specimen based on the stress release in the sample. Pre-tightening force makes the sample be easy to bend and causes the actual cutting quantity to increase. that is to say superposition of small deformation and the clamping limited bending deformation, then lead to deviation of theories and actual measured data. Secondly, the calculate principle of LRM is to suppose that each layer's depth is $t$, although it is difficult to make it, ideal deformation can be acquired by the finite element simulation, and fixed the deformation error of them. As a result of the vice clamping force is still in the elastic range, actual deformation amount can be acquired by using the principle of superposition.

The experimental and simulation conditions. Taking $7075 \mathrm{~T} 6$ rolling thick plate, size of $1200 \mathrm{~mm} \times 220 \mathrm{~mm} \times 50 \mathrm{~mm}$, making solute heat treatment and pre-stretching, in which temperature is $475^{\circ} \mathrm{C}$ and cooling in $15^{\circ} \mathrm{C}$ water. Pre-stretching operates in stretch tool of $5 \mathrm{MN}$.

Simulation of quenching and cutting by using MSC.Marc ${ }^{\circledR}$ involves a coupled thermo-mechanical analysis that ignores phase transformation effects. Size and characters of 
research object is same between simulation and experimental materials, i.e.7075 aluminum alloy rolling plates. The size of plate is $1200 \mathrm{~mm}(\mathrm{~L}) \times 220 \mathrm{~mm}(\mathrm{~W}) \times(30 \sim 50) \mathrm{mm}(\mathrm{H})$ and heat treatment is at $475^{\circ} \mathrm{C}$ for $120 \mathrm{~min}+15^{\circ} \mathrm{C}$ cooling nature aging. The shape of specimen surface is square. There are hypothesis be adopted that the material is isotropy and continuous medium, and the inner stress is symmetrical distribution through depth according to quenching condition.

The error analysis. Samples are $160 \mathrm{~mm}$ and $120 \mathrm{~mm}$ square with thickness of $30 \mathrm{~mm}$ plates, which are cut out in the water-bath quenching simulation model, and "life-death element technique" is used to simulate the milling process. Fig. 1 is the boundary conditions of milling model, and vice clamping force is about $20 \mathrm{MPa}$, the deformation is measured per milling depth of $2 \mathrm{~mm}$.

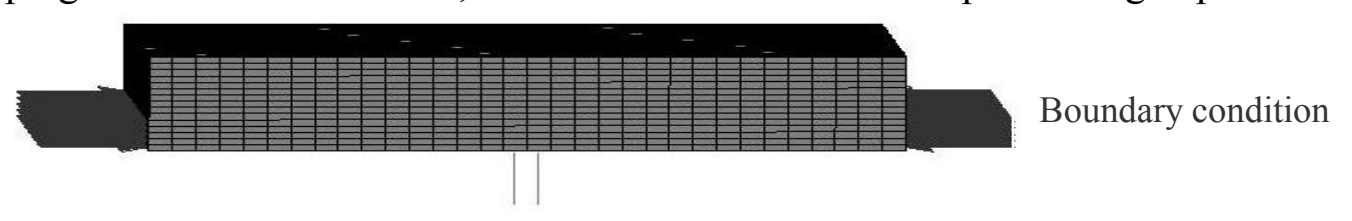

Fig.1 Milling clamping model of sample

Fig. 2 Is the deformation process of the samples $120 \times 120 \times 30(\mathrm{~mm})$ after milling. Obviously, thickness of the plate is not change.

(a) Sample geometry and distribution of initial stress

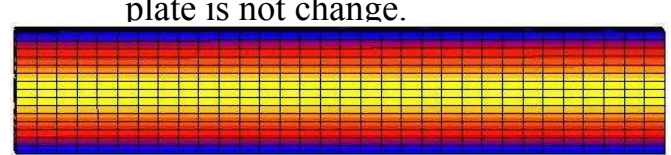

(b) deformation of sample after $3 \times 2 \mathrm{~mm}$ layer removed

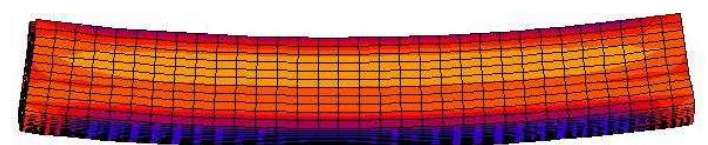

(c) deformation of sample after $7 \times 2 \mathrm{~mm}$ layer removed

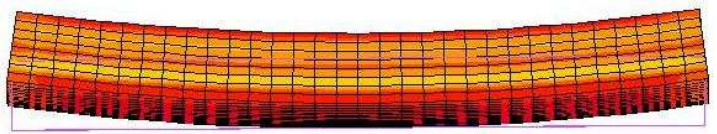

Fig.2 Diagrammatic sketch of specimen clamping deformation in the simulation process

However, the actual milling situation as shown in Fig. 3, in which the both ends are thin while the middle is thick as a result compared with deformation of Fig.2. Loosen the clamp, the sample will be bending, but the bending deformation will increase after measured. Again and again, the deformation error presents the shape of sample. So we can find the experiment of LRM exists inevitable error due to the processing behavior.

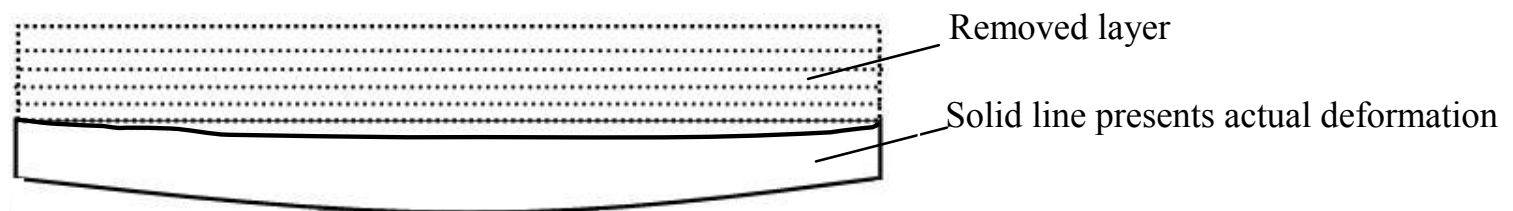

Fig.3 Diagrammatic sketch of specimen clamping deformation in the actual milling process

Table.1 Simulation-experimental deformation deviation in the milling process

\begin{tabular}{|c|c|c|c|c|c|c|c|}
\hline layer removal time & 1 & 2 & 3 & 4 & 5 & 6 & 7 \\
\hline Simulation $u \varepsilon$ & 408 & 795 & 1126 & 1460 & 1762 & 2040 & 2281 \\
\hline 160 actual measurement $u \varepsilon$ & 408 & 840 & 1250 & 1630 & 1930 & 2240 & 2490 \\
\hline Deviation ratio & $0 \%$ & $6 \%$ & $11 \%$ & $10 \%$ & $10 \%$ & $10 \%$ & $9 \%$ \\
\hline 120 actual measurement $u \varepsilon$ & 460 & 810 & 1200 & 1545 & 1850 & 2120 & 2360 \\
\hline Deviation ratio & $13 \%$ & $2 \%$ & $7 \%$ & $6 \%$ & $5 \%$ & $4 \%$ & $3 \%$ \\
\hline
\end{tabular}


Table. 1 reflects the difference of deformation under two conditions. The two sample deformations acquired by simulation are the same, however, the measured data has significant difference that the deviation of $160 \mathrm{~mm}$ square sample is about $10 \%$ and the deformation of $120 \mathrm{~mm}$ square specimen is about $6 \%$.Obviously, in layer removal experiments, the deformation data is larger caused by the bending deformation. At the same time, the deformation error of big sample is larger than small sample, which is associated with whether it is ease to deform or not. Certainly, Because of the difference of the actual clamping force every time, the percentage of deviation are different with the milling depth and specimen size. The experimental data show that, because of the deviation is not stable, we can try to describe this deviation distribution using a fitting function which can be used to calculate the results of compensation. This method is relatively more practical and simple.

In conclusion, based on the layer removal principle, the calculation of stress field need to be revised. Due to the superposition effect of small deformation and clamping deformation, the specific methods is that it can be approximated to fitting function based on the simulation and experiment deviation, and makes the experimental calculation be close to the simulation calculation.
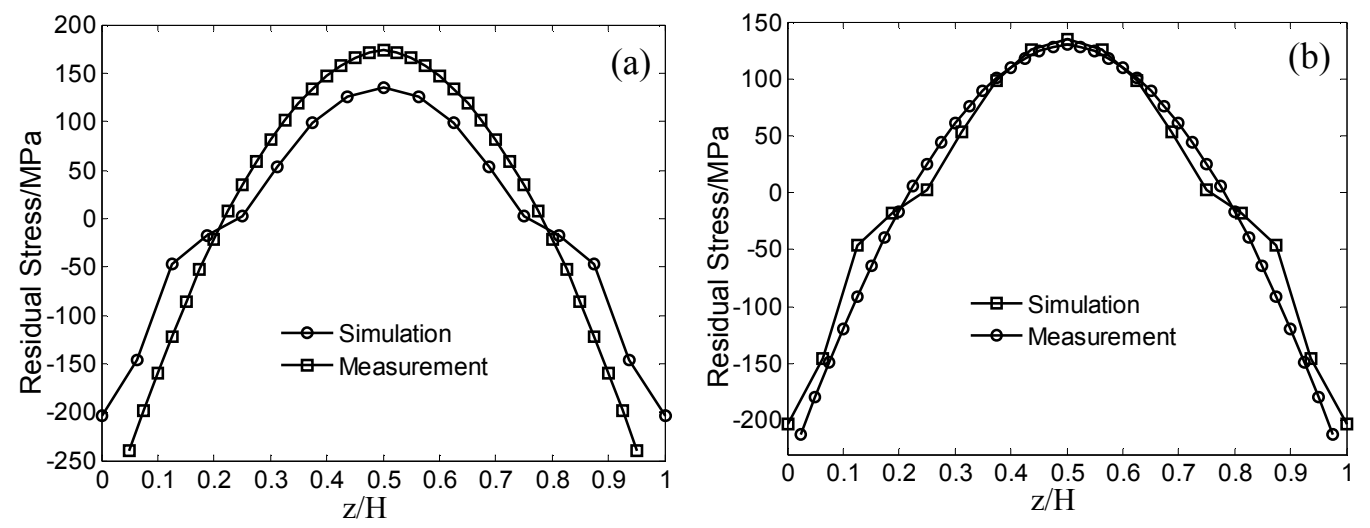

Fig.4 Calculation of experimental-simulation stress (a)before amended and (b)after amended

Under the same experimental conditions, $160 \mathrm{~mm}$ square sample removed from quenched plate and acquires stress field distribution curve after LRM experiments as shown in Fig.4(a), which presents the comparison between calculation and simulation results. Fig.4(b) shows the fitting function is well compensate for computation and the simulation are in good agreement with the experimental.

Amended result. Stress from both corresponding depth values are compared, and then acquired the approximate proportional correction of two results shown as Table.2。

Table. 2 Simulation-experiment stress deviation

\begin{tabular}{|c|c|c|c|c|c|c|c|c|}
\hline Layer depth $\mathrm{mm}$ & 1 & 3 & 5 & 7 & 9 & 11 & 13 & 15 \\
\hline Simulation $\mathrm{MPa}$ & -173 & -95 & -32 & -6 & 28 & 75 & 110 & 134 \\
\hline Calculation $\mathrm{MPa}$ & -240 & -121 & -43 & -5 & 34 & 101 & 134 & 170 \\
\hline Amended proportion & $28 \%$ & $21 \%$ & $25 \%$ & $20 \%$ & $17 \%$ & $25 \%$ & $17 \%$ & $21 \%$ \\
\hline
\end{tabular}

According to the different depth, the average compensation coefficient can be acquired along its depth, and then construct an amended function $f(z)$ through the method of data fitting approximation. As shown in Fig.5, the original deviation distribution is discrete, based on the 3 polynomial fitting, so an approximate curve of deviation can be shown. The amended function is constructed as follows. 


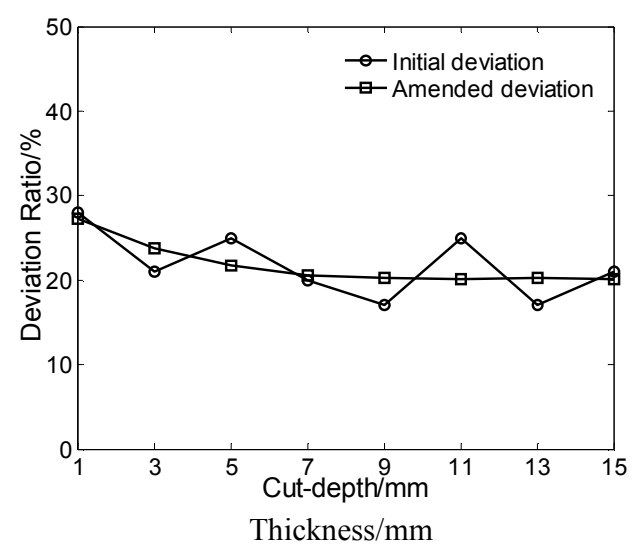

Fig.5 Fitting force deviation of simulation-experiment in $30 \mathrm{~mm}$ thick plate

$$
\begin{aligned}
& f(z)=\sum_{i=0}^{3} K_{i} z^{i} \\
& \sigma_{x}(z)=f(z) \cdot \sum_{i=0}^{n} A_{i} P_{i} ; \sigma_{y}(z)=f(z) \cdot \sum_{j=0}^{m} \mathrm{~B}_{j} P_{j}
\end{aligned}
$$

$K_{0}=-0.0063 ; K_{1}=0.22 ; \quad K_{2}=-2.5 ; \quad K_{3}=29.5$

The amended function is based on the following considerations: 1) Simulation and experiment is a kind of relative testing method. The stress determination and described are acquired in different ways, so taking the average of the two which can reflects propagation distribution. 2) There are deviation between experiment and simulation, which is due to the deformation caused by the superposition effect of accumulation. On the other hand, it is also due to the existence of certain differences in the node values of model fitting curve and experimental curve, so amended function is better able to balance this kind of error.
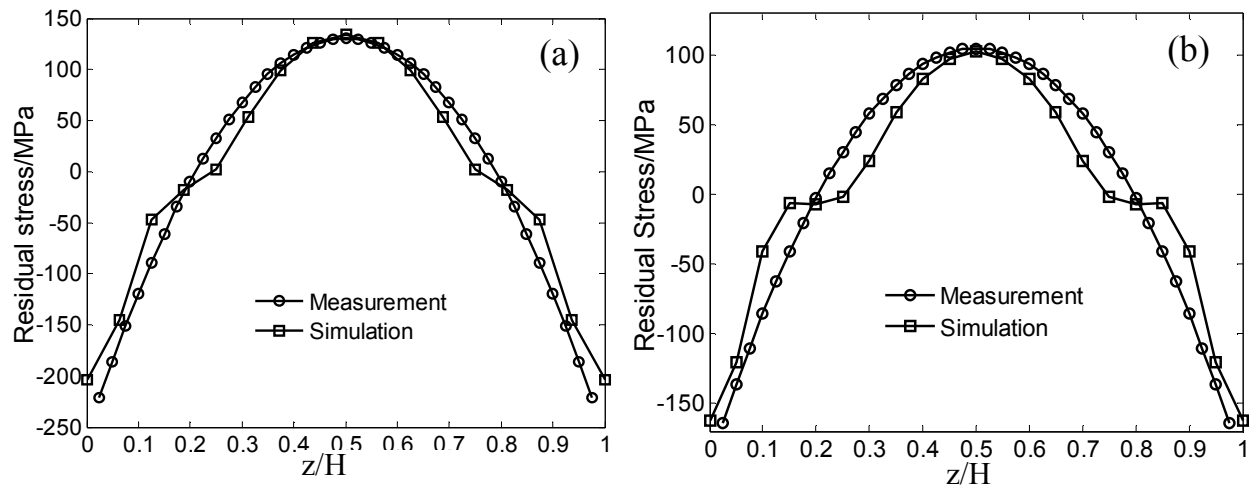

Fig.6 The amended experimental stress calculation and simulation results distribution

(a) $30 \mathrm{~mm}$ thick plate (b) $40 \mathrm{~mm}$ thick plate

Amended method for other plate experiments under the same conditions, also have good amended effect. Under the same experimental conditions, taking the $120 \mathrm{~mm}$ square sample of $30 \mathrm{~mm}$ plate and the $160 \mathrm{~mm}$ square sample of $40 \mathrm{~mm}$ plate, the comparison results between the simulation and the experiments as shown in Fig.6.

\section{Summary}

Based on the finite element simulation and experiment, the amended method to cumulative error of clamping deformation is proposed, and the right amended function is constructed, which is effective to solve the problem of experimental stress calculation. Amended function not only points out the experiment error of layer removal method, but also amends the experimental calculation model, which makes the results more reasonable. 


\section{Acknowledgment}

This work was funded by Guangxi Natural Science Foundation, China (No. 2012GXNSFBA053150), Open Research Fund of Key Laboratory of High Performance Complex Manufacturing(Central South University), China (No. HPCM-2013-04) and the Science and Technology Project of Hunan Province, China (No. 2014FJ3002)

\section{References}

[1] D. J. Greving. Residual stresses and thermal spray coating performance: Ph.D. Unite States: The University of Tulsa, 1995.

[2] Gong H, Wu Y X, Liao K. Influence of pre-stretching on residual stress distribution in 7075 aluminum alloy thick-plate[J]. Transactions of Materials and Heat Treatment. 30(2010)201-205.

[3] GUO Huai, ZuoDun-wen, Wang Shu-hong, WangMin. Measurement of Residual Stress Distribution in Thick Pre-Stretched Aluminum Alloy Plate. Journal of South China University of Technology.34(2006)33-36.

[4] M. B. Prime, Michael R. Hill. Uncertainty analysis, Model Error, and Order Selection for Series-Expanded, Residual-Stress Inverse Solutions[J]. Journal of Engineering Materials and Technology. 11(2006)175-185.

[5] Virkar, A. V. Determination of Residual Stress Profile Using a Strain Gage Technique. Journal of the American Ceramic Society.73(1990)2100-2102.

[6] Gary S. Schajer, Michael B. Prime. Use of inverse solutions for residual stress measurement. Journal of Engineering Materials and Technology, 128(2006)375-382.

[7] LIAO K. Research on Mechanism and Measurement Method of Quenching-Prestretching Stress in Aluminum Alloy Thick Plate: Ph.D. China: Central South University, 2010. 